University of Nebraska - Lincoln

DigitalCommons@University of Nebraska - Lincoln

7-20-1987

\title{
Observation of steepening in electron plasma waves driven by stimulated Raman backscattering
}

Donald Umstadter

University of Nebraska-Lincoln, donald.umstadter@unl.edu

R. Williams

University of California, Los Angeles

C. Clayton

University of California, Los Angeles

C. Joshi

University of California, Los Angeles

Follow this and additional works at: https://digitalcommons.unl.edu/physicsumstadter

Part of the Physics Commons

Umstadter, Donald; Williams, R.; Clayton, C.; and Joshi, C., "Observation of steepening in electron plasma waves driven by stimulated Raman backscattering" (1987). Donald Umstadter Publications. 35.

https://digitalcommons.unl.edu/physicsumstadter/35

This Article is brought to you for free and open access by the Research Papers in Physics and Astronomy at DigitalCommons@University of Nebraska - Lincoln. It has been accepted for inclusion in Donald Umstadter Publications by an authorized administrator of DigitalCommons@University of Nebraska - Lincoln. 


\title{
Observation of Steepening in Electron Plasma Waves Driven by Stimulated Raman Backscattering
}

\author{
D. Umstadter, R. Williams, C. Clayton, and C. Joshi \\ University of California, Los Angeles, Los Angeles, California 90024
}

(Received 5 February 1987)

\begin{abstract}
Harmonics of plasma waves excited by $\mathrm{CO}_{2}$-laser-induced stimulated Raman scattering have been observed by frequency- and wave-number-resolved ruby Thomson scattering. Measurements of their relative amplitudes agreed with predictions of nonlinear warm-plasma wave-steepening theory up to the maximum observed amplitude of the fundamental component, $\tilde{n}_{1} / n_{0}=16 \%$.
\end{abstract}

PACS numbers: $52.35 . \mathrm{Mw}, 52.35 . \mathrm{Fp}, 52.40 . \mathrm{Nk}$

A basic prediction of nonlinear plasma-wave theory is that as an electron plasma wave grows its waveform steepens. Equivalently, the harmonic components of the density perturbation increase in amplitude relative to the fundamental. This process has several implications beyond its relevance to basic plasma physics. ${ }^{1}$ It may, for instance, allow the plasma wake-field accelerator to exceed the linear transformer ratio. ${ }^{2}$ The presence of the second harmonic also strongly affects the time evolution of the fundamental in numerical investigations of a beam-plasma system. ${ }^{3}$ Steepening may also reduce Raman backscatter (SRS), an instability that is of current interest in laser fusion ${ }^{4}$ because it may hinder the absorption of laser energy by the plasma. An electrostatic plasma oscillation is excited in SRS by the ponderomotive force due to the beating between an incident electromagnetic wave and a light wave that is reflected in the backward direction from plasma noise. By reflecting light more strongly, it further enhances the ponderomotive force and both it and the reflected wave grow exponentially. Unless it saturates or its pump becomes depleted, the plasma wave eventually breaks. ${ }^{1}$ This is thought to be the origin of hot electrons that may preheat the fuel in laser fusion and thereby reduce compression efficiency. ${ }^{5}$

In this paper we report the direct experimental observation of steepening in plasma waves driven by SRS. In particular, we show for the first time that the amplitudes of the first three harmonics were consistent with the nonlinear theory of warm-plasma-wave growth. ${ }^{1}$

Electron plasma-wave steepening was first predicted by Dawson. ${ }^{1}$ An expansion of the steepened density perturbation in terms of amplitudes of harmonic components was found with the use of the fluid equations and the assumption of a cold plasma by Jackson. ${ }^{1}$ These same amplitudes may be found for the specific case of plasma waves driven by SRS from Koch and Albritton. ${ }^{1}$ They may be expressed in terms of the fundamental $\left(\tilde{n}_{1} / n_{0}\right)$ as $\tilde{n}_{m} / n_{0}=\alpha_{m}\left(\tilde{n}_{1} / n_{0}\right)^{m}$, where $m$ is an integer $\geq 1, \quad \alpha_{m}($ cold $)=m^{m / 2}{ }^{(m-1)} m !$, and $\tilde{n}_{m} / n_{0}$ is the fluctuating density of the $m$ th harmonic normalized to the background density. The coefficients for the first two higher harmonics are $\alpha_{2}($ cold $)=1.0$ and $\alpha_{3}($ cold $)=1.1$.
These coefficients were rederived by use of the kinetic equations on the assumption of a warm plasma, by Kuz'menkov et al. ${ }^{1}$ They are $\alpha_{2}$ (warm) $=1.3$ and $\alpha_{3}($ warm $)=1.9$ for the conditions of our experiment ( $T_{e}=10 \mathrm{eV}$ and $v_{\phi} / c=0.02$, where $v_{\phi}$ is the phase velocity). Of course, the kinetic treatment agrees with the cold-plasma treatment in the cold-plasma limit $\left(T_{e}=0\right)$. It also predicts a nonlinear frequency shift, which for our experimental conditions is $\simeq 0.3 \%$, less than the resolution of our spectrometer.

The incident $\left(\omega_{0}, k_{0}\right)$, reflected $\left(\omega_{1}, k_{1}\right)$, and plasma $\left(\omega_{2}, k_{2}\right)$ waves in SRS must obey their dispersion relations, $\omega_{0,1}^{2}=\omega_{p}^{2}+c^{2} k_{0,1}^{2}$ and $\omega_{2}=\omega_{p}\left[1+3\left(k_{2} \lambda_{D}\right)^{2}\right]^{1 / 2}$, and the phase matching conditions, $\omega_{0}=\omega_{1}+\omega_{2}$ and $\mathbf{k}_{0}=\mathbf{k}_{1}+\mathbf{k}_{2}$, where $\lambda_{\mathrm{D}}=\left(k T_{e} / m_{e} \omega_{p}^{2}\right)^{1 / 2}$ is the Debye length. Thus for Raman backscatter to occur in an underdense plasma $\left(n / n_{c} \equiv \omega_{p}^{2} / \omega_{0}^{2} \ll 1\right)$, the plasma-wave fundamental must have wave number $k_{2} \simeq 2 k_{0}$. We expect for the $m$ th harmonic that $(\omega, k)=\left(m \omega_{2}, m k_{2}\right)$.

Ion-acoustic waves may be excited by the stimulated Brillouin scattering (SBS) instability, which for our experimental parameters has a lower threshold than SRS. Since the ion-acoustic frequency $\omega_{a} \equiv k_{i} c_{s} \ll \omega_{0}$, where $c_{s}=\left(\gamma Z k T_{e} / m_{i}\right)^{1 / 2}$ is the acoustic velocity, the wave number of the ion-wave fundamental, $k_{i}$, also equals $2 k_{0}$. Harmonics of SBS-induced ion waves are observed by several groups. ${ }^{6}$

The plasma waves were excited in this experiment with a $10.6-\mu \mathrm{m} \mathrm{CO}_{2}$ laser $(10 \mathrm{~J}, 2 \mathrm{~ns})$, which was focused by an $f / 7.5$ lens to a spot of diameter $d \leq 300 \mu \mathrm{m}$ onto an arc-preionized $\mathrm{H}_{2}$ plasma. Raman backscattered light was diverted from the path of the incident $\mathrm{CO}_{2}$ beam (pump) with use of a beamsplitter. Its amplitude could then be analyzed as a function of time with a resolution of 250 ps with a liquid-He-cooled detector in combination with a grating spectrograph, or as a function of frequency by addition of an image dissector. ${ }^{7}$ The plasma waves were directly detected by Thomson scattering with use of a 1-J, 15-ns ruby laser pulse $(\lambda=0.6943 \mu \mathrm{m})$, which was synchronized in time with the $\mathrm{CO}_{2}$ pulse and brought transversely to a focus at the location of the $\mathrm{CO}_{2}$ beam waist. The amplitude of the reimaged Thomson-scattered light was analyzed as a 
function of frequency by use of an optical multichannel analyzer in tandem with a grating spectrograph. The frequency of the plasma wave was determined by the frequency shift, $\Delta \omega=\omega_{2}$, of either the SRS or the Thomson scattering. Its direction relative to the pump was determined by the sign of the frequency shift. Its wave number was determined by adjustment of the scattering angle $\left(\theta_{s}\right)$ between the incident beam and the collection lens for the Thomson-scattered beam. This angle is uniquely determined by the wave number of the plasma wave, $k_{2}$, by the $k$-matching condition, $\mathbf{k}_{\text {probe }}=\mathbf{k}_{s} \pm \mathbf{k}_{2}$, where $k_{\text {probe }}$ and $k_{s}$ are the wave numbers of the ruby probe and scattered beam, respectively.

Two narrow-frequency lines were observed when the collection lens for the ruby Thomson-scattered light was at $\theta_{s}=7^{\circ}$, the angle corresponding to $k_{2}=2 k_{0}$ (1 $\pm 15 \%$ ); the uncertainty was due to the $4.5^{\circ}$ angle subtended by the collection lens. The peak on the right of Fig. 1 (a), which shows a photograph of the optical multichannel analyzer output, is due to Thomson scattering from an SBS-induced ion wave. SBS was expected because the pump laser intensity, $I$, was well above its threshold. This feature was detected only when the $\mathrm{CO}_{2}$ laser was incident on the plasma and thus was not due to ruby stray light from specular reflections. The Thomson-scattered signal from the ion wave appears unshifted in frequency because the ion-acoustic frequency, $\omega_{a}$, was less than the resolution of the spectrometer. The acoustic frequency was, nevertheless, measured by use of a spectrometer with a greater resolution and in this way the electron temperature was determined to be $T_{e} \simeq 10$ eV. The peak on the left of Fig. 1(a) is due to Thomson-scattered light that is blue shifted in frequency
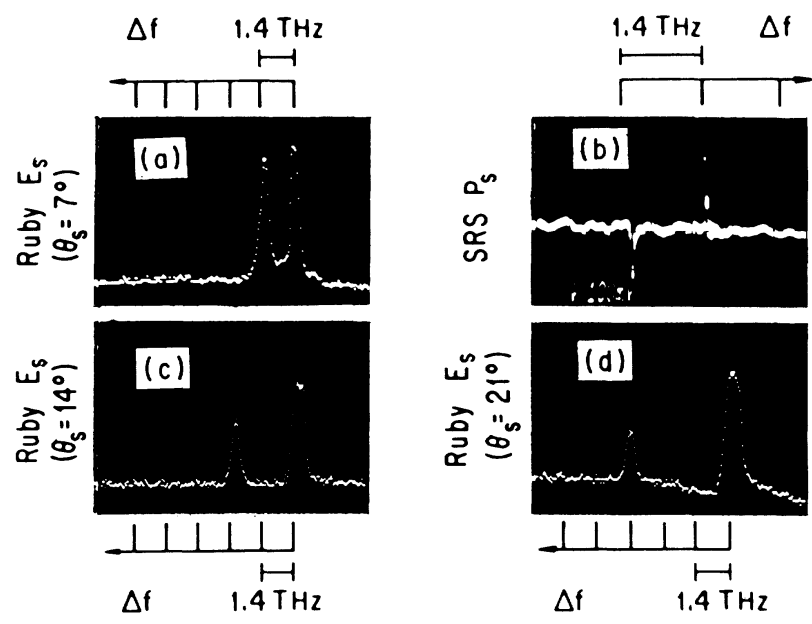

FIG. 1. Frequency and wave-number spectra of plasmawave harmonics. (a) Thomson-scattered light from the fundamental of the ion wave (right) and plasma wave (left). (b) Raman backscatter (right) and a fiducial at $10.6 \mu \mathrm{m}$ (inverted, left). (c) The second and (d) third harmonics of the ion wave (right) and the plasma wave (left).
$(\Delta f)$ by an amount that, with the assumption of $\Delta \omega=\omega_{2}$ and $T_{e}=10 \mathrm{eV}$, is consistent with a plasma density of 2.0 $\times 10^{16} \mathrm{~cm}^{-3}\left(n_{0} / n_{c}=2 \times 10^{-3}\right)$ and a value $k_{2} \lambda_{\mathrm{D}}=0.2$. Again, no peak was observed when the probe was fired without the $\mathrm{CO}_{2}$ laser. These results imply that this was not incoherent scattering from thermal fluctuations, but collective scattering $\left(k_{2} \lambda_{\mathrm{D}} \ll 1\right)$ from the fundamental component of a plasma wave that was propagating in the direction of the pump with $(\omega, k)=\left(\omega_{2}, k_{2}\right)$.

The peak on the right of Fig. 1(b), which shows an oscilloscope trace of the image-dissector output, is due to red-shifted SRS backscatter, measured simultaneously with the $7^{\circ}$ Thomson scattering [Fig. 1(a)]. The negative pulse on the left is a fiducial used to determine the frequency shift $(\Delta f)$ of the backscattered light with respect to $10.6 \mu \mathrm{m}$, the incident pump wavelength. By variation of the arc fill pressure and the relative timing between the preionization and the laser pulse, the plasma density could be continuously adjusted. As shown in Fig. 2(a), the densities calculated from the frequency shifts of both the SRS and the $7^{\circ}$ Thomson scattering agree over the density range of the experiment. We thus conclude that both were the decay products of the incident light wave; the backscatter was the daughter light wave and the Thomson scatter was scattering from the fundamental component of the daughter plasma wave.

We chose to operate at $n_{0} / n_{c}=2 \times 10^{-3}$; it was the density at which SRS and thus the plasma-wave ampli-

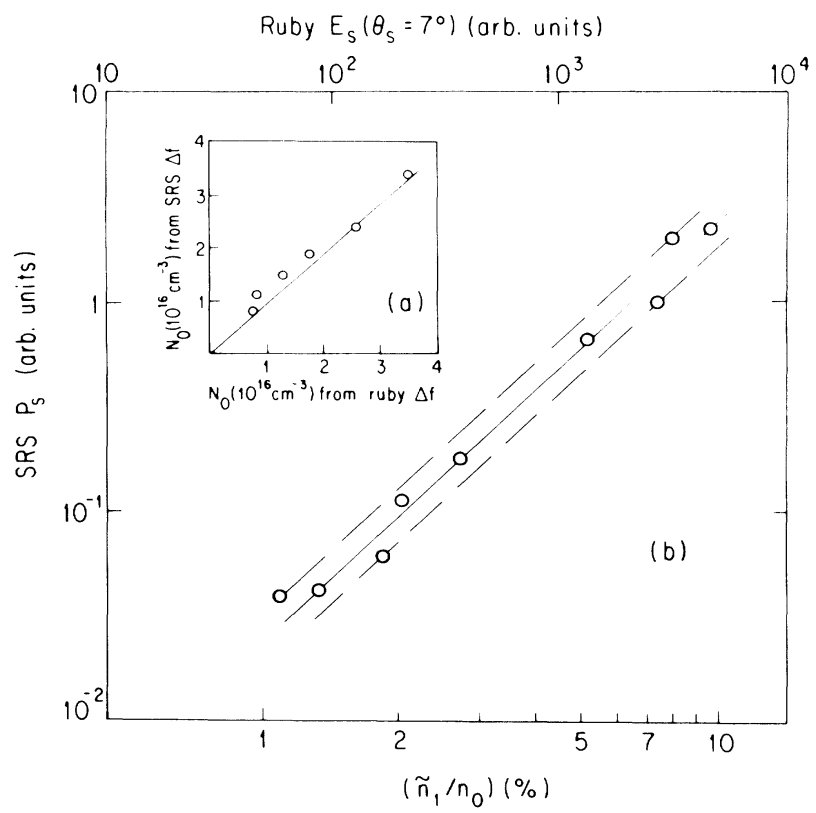

FIG. 2. (a) Density $\left(N_{0}\right)$ inferred from the ruby Thomsonscatter frequency shift, $\Delta f$, plotted with $N_{0}$ inferred from the SRS $\Delta f$. (b) Amplitude correlation of the ruby Thomson scatter $\left(E_{s}\right)$ with Raman backscatter $\left(P_{s}\right)$. The solid line is a best fit to the data and the dashed lines represent the spread in measured values. 
tude were maximum, possibly because of the competition between collisional damping at higher $n_{0}$ and Landau damping at lower $n_{0}{ }^{8}$

The peak on the left in Fig. 1(c)-which is due to ruby probe light scattered at $\theta_{s}=14^{\circ}$, the angle corresponding to $k_{2}=4 k_{0}(1 \pm 8 \%)$-is shifted in frequency by $\Delta \omega$ $=2 \omega_{2}$; that is twice the frequency shift of the probe light scattered at $\theta_{s}=7^{\circ}$ [Fig. 1(a)]. By monitoring of the shift in frequency of the SRS backscattered light [Figs. 1 (b) and 2(a)], it was found that the density, and thus the plasma-wave frequency, $\omega_{2}$, was the same for both $\theta_{s}=7^{\circ}$ and $\theta_{s}=14^{\circ}$ scattering. The peak on the left of Fig. 1(c), therefore, is due to scattering from the second-harmonic component of the plasma wave with $(\omega, k)=\left(2 \omega_{2}, 2 k_{2}\right)$. The peak on the right of Fig. 1 (c) is due to scattering from the second harmonic of the ion wave. Similarly, the two peaks in Fig. 1(d), corresponding to ruby light scattered at $\theta_{s}=21^{\circ}$, are attributed to the third-harmonic component of the plasma wave, with $(\omega, k)=\left(3 \omega_{2}, 3 k_{2}\right)$, and the third harmonic of the ion wave. Although harmonics of electron plasma waves excited in a discharge tube are observed, ${ }^{9}$ the results of Fig. 1 are, we believe, the first observation of harmonics of plasma waves driven by SRS.

The peak amplitudes of the backscattered power $\left(P_{s}\right)$ are plotted in Fig. 2(b) with the simultaneously measured energies $\left(E_{s}\right)$ of the $7^{\circ}$ Thomson-scattered light with $\Delta \omega=\omega_{2}$ [Fig. 1(a)]. The Bragg scattering formula, ${ }^{10}$

$$
\frac{E_{s} / \tau_{p}}{E_{0} / \tau_{0}} \simeq \frac{P_{s}}{P_{0}}=\frac{1}{4} \tilde{n}^{2} r_{0}^{2} \lambda_{\text {probe }}^{2} d^{2}
$$

where $r_{0}$ is the classical electron radius, allows conversion of $E_{s}\left(\theta_{s}=7^{\circ}\right)$ to $\tilde{n}_{1} / n_{0}$, if we assume an effective $d=120 \mu \mathrm{m},{ }^{11}$ or approximately one half of the width of the $\mathrm{CO}_{2}$ beam waist, ${ }^{11}$ and a ratio of the ruby pulse length to the lifetime of the plasma wave $\tau_{0} / \tau_{p}=(15$ $\mathrm{ns}) /(1.5 \mathrm{~ns})=10$. The latter was determined from measured time histories of both the ruby pulse $\left(\tau_{0}\right)$ and the backscattered pulse $\left(\tau_{p}\right)$. The scattering system was calibrated absolutely by direct measurement of the power of the incident probe beam, $P_{0}$, with the same optics and optical multichannel analyzer that was used to measure $P_{s}$.

As can be seen from Fig. 2(b), the maximum backscatter corresponds to a value of $\left(\tilde{n}_{1} / n_{0}\right)_{\mathrm{TS}}=10 \%$ for the electron plasma wave, measured by $\left(\theta_{s}=7^{\circ}\right)$ ruby Thomson scattering. This is consistent with a value of $9 \% \leq\left(\tilde{n}_{1} / n_{0}\right)_{\mathrm{SRS}}$, which is inferred from the amount of backscatter and a formula for the reflectivity, ${ }^{12}$

$$
R=\tanh ^{2}\left[\frac{\pi}{2} \frac{\tilde{n}_{1}}{n_{0}} \frac{n_{0}}{n_{c}} \frac{L}{\lambda_{\text {pump }}}\left(1-\frac{n_{0}}{n_{c}}\right)^{-1 / 2}\right],
$$

with the assumption of a value for $L$, the length of the interaction region, of $L / \lambda_{0} \leq 190 ; L$ was measured by our observing the level of Thomson scattering while scanning the plasma with the ruby probe along the axis of the $\mathrm{CO}_{2}$ laser focus and simultaneously monitoring the amplitude of the Raman backscatter. As can be seen by Eqs. (1) and (2), in the limit of small argument both SRS and Thomson scattering have the same dependence on $\tilde{n}_{1} / n_{0}$. The linear correlation of Fig. 2(b), therefore, indicates that not only the maximum value but all values of $\left(\tilde{n}_{1} / n_{0}\right)_{\mathrm{TS}}$ are consistent with the inferred range of $\left(\tilde{n}_{1} / n_{0}\right)_{\text {SRS. }}$.

The condition for ion-wave harmonic generation is $\left(k_{i} \lambda_{\mathrm{D}}\right)^{2}=\left(\tilde{n}_{1} / n_{0}\right)_{\text {ion. }}{ }^{6}$ From the amount of SBS and Eq. (1), $\tilde{n}_{1} / n_{0}$ for the ion wave was typically $\left(\tilde{n}_{1} / n_{0}\right)_{\text {ion }}$ $=10 \%$. Since $\left(k_{i} \lambda_{\mathrm{D}}\right)^{2}=4 \%$, ion-wave harmonics were thus to be expected.

By virtue of the correlation of Fig. 2(b), while Thomson scattering is used to measure either $\tilde{n}_{2} / n_{0}$ or $\tilde{n}_{3} / n_{0}$ for the electron plasma wave-but not both on the same shot - SRS can be used to infer $\tilde{n}_{1} / n_{0}$. The amplitude of the backscattered light is plotted in Fig. 3 with the amplitude of the light Thomson scattered at $\theta_{s}=14^{\circ}$ and $\theta_{s}=21^{\circ}$ from the second and third harmonics, respectively. The scale of the ordinate in Fig. 3 was changed from $E_{s}\left(\theta_{s}=14^{\circ}\right)$ to $\tilde{n}_{2} / n_{0}$ with use of $d=85 \mu \mathrm{m}$ and from $E_{s}\left(\theta_{s}=21^{\circ}\right)$ to $\tilde{n}_{3} / n_{0}$ with use of $d=70 \mu \mathrm{m}$. Here, assuming that the harmonics had Gaussian profiles and were related to the fundamental quadratically and cubically, respectively, we took $d$ to be the width at which the amplitude was reduced by $1 / e$ of its maximum value. The scale of the abscissa was determined by use of the correlation that is represented by the solid line in Fig. 2 (b) to infer $\tilde{n}_{1} / n_{0}$ from the SRS amplitude. The predictions of the warm-plasma theory, represented by the solid lines in Fig. 3, were obtained with $\left(\tilde{n}_{2} / n_{0}\right)_{\text {pred }}$

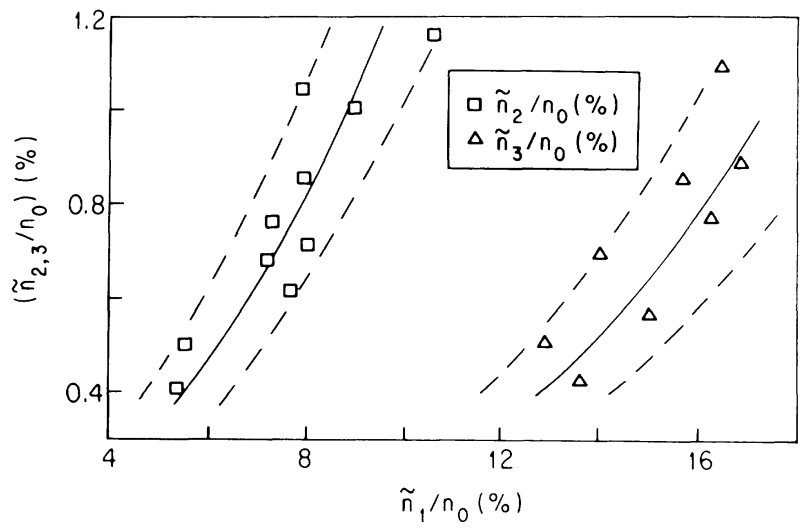

FIG. 3. Amplitude of Thomson scattering from the second harmonic $\left(\tilde{n}_{2} / n_{0}\right)$ and third harmonic $\left(\tilde{n}_{3} / n_{0}\right)$ as a function of Raman backscatter $\left(\tilde{n}_{1} / n_{0}\right)$. The solid lines represent $\left(\tilde{n}_{2} /\right.$ $\left.n_{0}\right)_{\text {pred }}$ (left) and $\left(\tilde{n}_{3} / n_{0}\right)_{\text {pred }}$ (right). The dashed lines represent the uncertainty in $\left(\tilde{n}_{2} / n_{0}\right)_{\text {pred }}$ or $\left(\tilde{n}_{3} / n_{0}\right)_{\text {pred, due to }}$ the uncertainty in $\tilde{n}_{1} / n_{0}$. 
$=1.3\left(\tilde{n}_{1} / n_{0}\right)^{2}$ and $\left(\tilde{n}_{3} / n_{0}\right)_{\text {pred }}=1.9\left(\tilde{n}_{1} / n_{0}\right)^{3}$. The uncertainties in $\left(\tilde{n}_{2} / n_{0}\right)_{\text {pred }}$ and $\left(\tilde{n}_{3} / n_{0}\right)_{\text {pred }}$, represented by the dashed lines in Fig. 3, were due to the uncertainty in $\tilde{n}_{1} / n_{0}$, represented by the dashed lines of Fig. 2(b). The data of Figs. 2(b) and 3 were obtained on separate data runs. There are values of $P_{s}$ in Fig. 3 that are larger than its maximum value in Fig. $2(\mathrm{~b}), P_{s}\left(\tilde{n}_{1} / n_{0}=10 \%\right)$. The curve of Fig. 2(b), therefore, had to be extrapolated to the range $10 \%<\tilde{n}_{1} / n_{0} \leq 16 \%$. This was justified not only because of the correlation at lower values but because both Eqs. (1) and (2) depend only on $\tilde{n}_{1} / n_{0}$. For all values shown in Fig. 3, the measured value of $\tilde{n}_{2} / n_{0}$ agrees with $\left(\tilde{n}_{2} / n_{0}\right)_{\text {pred }}$, and $\tilde{n}_{3} / n_{0}$ agrees with $\left(\tilde{n}_{3} /\right.$ $\left.n_{0}\right)_{\text {pred. }}$.

In summary, we have observed the harmonics of plasma waves driven by SRS and found their frequencies, wave numbers, and amplitudes to be consistent with warm-plasma wave-steepening theory.

We thank Dr. T. Katsouleas, Dr. F. F. Chen, Dr. J. Dawson, Dr. W. Mori, and Dr. H. Figueroa for many useful discussions. This work was supported by the U.S. Department of Energy Grant No. DE-AS03-83-ER40120, National Science Foundation Grant No. ECS810972, and the Lawrence Livermore National Laborato- ry University Research Program.

'J. M. Dawson, Phys. Rev. 113, 383 (1959); E. A. Jackson, Phys. Fluids 3, 831 (1960); P. Koch and J. Albritton, Phys. Rev. Lett. 34, 1616 (1975); L. S. Kuz'menkov, A. A. Sokolov, and O. O. Trubachev, Izv. Vyssh. Uchebn. Zaved. Fiz. 12, 17 (1983) [Sov. Phys. J. 26, 1076 (1984)].

${ }^{2}$ J. Rosenweig, Phys. Rev. Lett. 58, 555 (1987).

${ }^{3}$ J. P. Klozenberg, Phys. Fluids 14, 94 (1971).

${ }^{4}$ G. McIntosh, H. Houtman, and J. Meyer, Phys. Rev. Lett. 57, 337 (1986), and references quoted therein.

${ }^{5}$ W. L. Kruer et al., Phys. Fluids 23, 1326 (1980).

${ }^{6}$ C. J. Walsh and H. A. Baldis, Phys. Rev. Lett. 48, 1483 (1982); C. Clayton, C. Joshi, and F. F. Chen, Phys. Rev. Lett. 51, 1656 (1983).

${ }^{7}$ H. A. Baldis, N. H. Burnett, and M. C. Richardson, Rev. Sci. Instrum. 48, 173 (1977).

${ }^{8}$ R. G. Watt, R. D. Brooks, and Z. A. Pietrzyk, Phys. Rev. Lett. 41, 170 (1978).

${ }^{9}$ T. Kawabe, S. Kojima, and K. Takayama, J. Phys. Soc. Jpn 20, 1539 (1965).

${ }^{10}$ R. E. Slusher and C. M. Surko, Phys. Fluids 23, 472 (1980).

${ }^{11}$ C. E. Clayton, C. Joshi, C. Darrow, and D. Umstadter, Phys. Rev. Lett. 54, 2343 (1985).

${ }^{12}$ W. L. Kruer, Phys. Fluids 23, 127 (1980). 